\title{
Controlling active self-assembly through broken particle-shape symmetry
}

\author{
H. H. Wensink, ${ }^{1, *}$ V. Kantsler, ${ }^{2}$ R. E. Goldstein, ${ }^{2}$ and J. Dunkel ${ }^{2,3}$ \\ ${ }^{1}$ Laboratoire de Physique des Solides, Université Paris-Sud and CNRS, Bâtiment 510, 91405 Orsay Cedex, France \\ ${ }^{2}$ DAMTP, Centre for Mathematical Sciences, University of Cambridge, Wilberforce Road, Cambridge CB3 OWA, United Kingdom \\ ${ }^{3}$ Department of Mathematics, Massachusetts Institute of Technology, 77 Massachusetts Avenue, Cambridge, Massachusetts 02139, USA
}

(Received 1 June 2013; published 28 January 2014)

\begin{abstract}
Many structural properties of conventional passive materials are known to arise from the symmetries of their microscopic constituents. By contrast, it is largely unclear how the interplay between particle shape and self-propulsion controls the meso- and macroscale behavior of active matter. Here we use large-scale simulations of homo- and heterogeneous self-propelled particle systems to identify generic effects of broken particle-shape symmetry on collective motion. We find that even small violations of fore-aft symmetry lead to fundamentally different collective behaviors, which may facilitate demixing of differently shaped species as well as the spontaneous formation of stable microrotors. These results suggest that variation of particle shape yields robust physical mechanisms to control self-assembly of active matter, with possibly profound implications for biology and materials design.
\end{abstract}

DOI: 10.1103/PhysRevE.89.010302

PACS number(s): 82.70.Dd, 89.75.Kd

Introduction. Physical and chemical properties of conventional materials depend critically on the symmetries of their microscopic constituents [1-5]. The perhaps best known examples are carbon allotropes [6], such as diamond or graphite, which exhibit vastly different elastic and optical characteristics reflecting the tetrahedral or planar structure of their elementary building blocks. While the relation between microscopic symmetries and macroscopic properties of passive materials has been intensely studied both experimentally $[4,7,8]$ and theoretically [1-3,9], comparatively little is known about how constituent shape affects structure formation and collective motion in active matter [10-16]. Rapid progress in the fabrication of colloids [17] and Janus particles [18] with broken spherical [19] and cylindrical [20] symmetries [Fig. 1(a)] suggests the need for systematic theoretical studies to help identify generic principles for the design and selfassembly of novel active matter states. Additional biophysical motivation stems from the still limited understanding about the role of cell shape [21,22] in the evolution of multicellular behavior [12,23]. Although the relative importance of chemical, hydrodynamic, and steric interactions for collective microbial motion is still under debate [24,25], recent experiments [26] have shown that the different surface-scattering laws of sperm and Chlamydomonas cells arise from their effective dynamical shapes, that is, the volumes swept by their cilia during swimming [Figs. 1(b) and 1(c)]: Sperms can be viewed as forward-swimming cones that tend to accumulate at surfaces since, after an aligning collision, their velocity vector points into the boundary. By contrast, biflagellate Chlamydomonas algae resemble backward-swimming cones, departing from lateral boundaries at a narrowly distributed angle set by the ciliary beat [26]. These observations raise the question whether

\footnotetext{
*wensink@1ps.u-psud.fr
}

Published by the American Physical Society under the terms of the Creative Commons Attribution 3.0 License. Further distribution of this work must maintain attribution to the author(s) and the published article's title, journal citation, and DOI. similar shape-induced steric effects suffice to explain various types of collective dynamics in wet and dry active systems.

Here we show that even small violations of fore-aft symmetry may lead to fundamentally different modes of active collective motion. By analyzing large-scale simulations of two-dimensional (2D) self-propelled particles (SPPs), we find that the effective particle shape can be used to control the transition between two distinctly different collective behaviors commonly encountered in active systems: frontlike cooperative motion [27,28] and formation of active droplets [29] resembling multicellular colonies. These qualitatively different behaviors facilitate spontaneous demixing of inhomogeneous

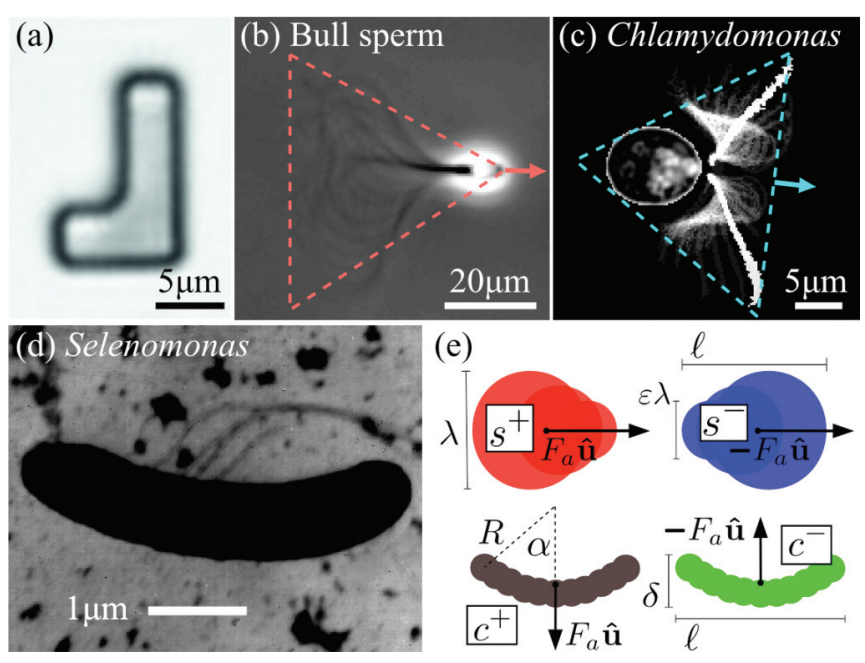

FIG. 1. (Color) Actual and effective dynamical shapes of various microswimmers and simplified representation in the SPP model. (a) An L-shaped self-driven colloid [20]. (b) Superimposed phasecontrast micrographs (Zeiss Axiovert, 40×, NA0.6) of swimming bull sperm. On time scales larger than the beat period $\sim 0.1 \mathrm{~s}$, the cell mimics a forward-swimming cone. (c) A Chlamydomonas alga $(63 \times$, NA1.3), confined to quasi-2D motion, resembles a backwardswimming triangle. (d) Nonconvex crescent-shaped Selenomonas bovis bacterium with flagella. (e) The SPP model approximates different shapes by combinations of rigidly linked spheres. 
systems, suggesting that the combination of particle shape and self-propulsion might have been a relevant evolutionary factor and also offering robust generic tuning mechanisms for the self-assembly of active materials. To illustrate the latter fact, we will demonstrate that nonconvex SPPs [Figs. 1(d) and 1(e)] can self-assemble into active rotors.

Model. We simulate $N$ SPPs in two dimensions, each driven by a constant self-propulsion force $F_{a}$ of fixed direction in the body frame. The 2D case is practically relevant as micro-organisms, colloids, or granules often experience strong geometric confinement such as solid surfaces and interfaces [26,30,31]. Focusing on two important classes of shapes, we compare convex polar (spermlike) SPPs $\left(s^{+}\right)$with antipolar SPPs $\left(s^{-}\right)$and nonconvex crescent-shaped $c^{+}$SPPs with anticrescents $\left(c^{-}\right)$, as defined in Fig. 1(e). Particles are assumed to move in the overdamped low-Reynolds-number regime [32], interacting with each other only by steric repulsion. Interparticle forces and torques are calculated by discretizing each SPP into $i=1, \ldots, n$ equidistant spherical segments with effective diameter $\lambda^{i}$. Defining $\lambda_{\alpha \beta}^{i j}=\left(\lambda_{\alpha}^{i}+\lambda_{\beta}^{j}\right) / 2$, the total pair potential $U_{\alpha \beta}=n^{-2} \sum_{i, j=1}^{n} u\left(r_{\alpha \beta}^{i j} / \lambda_{\alpha \beta}^{i j}\right)$ of two SPPs $\alpha$ and $\beta$ depends on their orientation unit vectors $\left\{\hat{\mathbf{u}}_{\alpha}, \hat{\mathbf{u}}_{\beta}\right\}$ and center-of-mass distance $\Delta \mathbf{r}_{\alpha \beta}=\mathbf{r}_{\alpha}-\mathbf{r}_{\beta}$ through the segment distance $r_{\alpha \beta}^{i j}=\left|\Delta \mathbf{r}_{\alpha \beta}+\mathbf{e}_{\alpha}^{i}-\mathbf{e}_{\beta}^{j}\right|$, where the vectors $\mathbf{e}_{\alpha}^{i}$ denote the position of segment $i$ relative to the mass center $\mathbf{r}_{\alpha}$ with respect to the body frame. Throughout, we adopt a repulsive short-range potential $u(x)=u_{0} \exp (-x) / x^{2}$ with amplitude $u_{0}>0$ and consider minimal deterministic equations of motion for the positions $\mathbf{r}_{\alpha}(t)$ and orientations $\hat{\mathbf{u}}_{\alpha}(t)=\left\{\sin \varphi_{\alpha}(t), \cos \varphi_{\alpha}(t)\right\}$ by balancing active and steric forces and torques,

$$
\mathbf{f}_{T} \cdot \partial_{t} \mathbf{r}_{\alpha}=-\nabla_{\mathbf{r}_{\alpha}} U+F_{a} \hat{\mathbf{u}}_{\alpha}, \quad \mathbf{f}_{R} \cdot \partial_{t} \varphi_{\alpha}=-\nabla_{\varphi_{\alpha}} U,
$$

where $U=\frac{1}{2} \sum_{\alpha, \beta(\alpha \neq \beta)} U_{\alpha \beta}$ is the total potential energy. The one-particle translational and rotational friction tensors $\mathbf{f}_{T}$ and $\mathbf{f}_{R}$ can be decomposed into parallel, perpendicular, and rotational contributions that depend solely on the aspect ratio $a$ for which we specify below effective values depending on the SPP shape [33]. Equation (1) neglects thermal or intrinsic Brownian noise [24] and hydrodynamic interactions, which is acceptable at intermediate-to-high concentrations when particle collisions dominate the dynamics [12,24] and when nearby no-slip boundaries suppress hydrodynamic forces.

We integrated Eq. (1) numerically using a square simulation box (area $A$ ) with periodic boundary conditions. The initial condition was a cubic SPP lattice with motility directions chosen randomly. In simulations with more than one species, particles were randomly distributed on the lattice. The distance between neighboring SPP segments was kept small to avoid crossing of SPPs [Fig. 1(e)]. To reduce the number of parameters in simulations, Eq. (1) was rewritten in terms of the dimensionless time $\tau=t v_{0} / \lambda$, adopting the largest segment diameter $\lambda$ as the length unit and the self-propulsion speed $v_{0}=F_{a} /\left\|\mathbf{f}_{T}\right\|$ of a noninteracting SPP as the velocity unit. The rescaled potential amplitude $\tilde{u}_{0}=u_{0} / F_{a} \lambda$ has little or no effect on the collective behavior as long as $\tilde{u}_{0}>10$ and the SPP dynamics is governed by the volume fraction $\phi=N \sigma / A$, with $\sigma$ the area per particle, and the intrinsic shape parameters of the SPPs (aspect ratio, polarity, curvature, etc.).
Convex SPPs. We first consider convex polar $s^{+}$SPPs and antipolar $s^{-}$SPPs [Fig. 1(e)], composed of $n=3$ equidistant spherical segments ${ }^{1}$ and representing prototypical polar SPPs with broken fore-aft symmetry, $U_{\alpha \beta}\left(\hat{\mathbf{u}}_{\alpha}, \hat{\mathbf{u}}_{\beta}\right) \neq U_{\alpha \beta}\left(\hat{\mathbf{u}}_{\alpha},-\hat{\mathbf{u}}_{\beta}\right)$. The geometric polarity $p=\left(\lambda^{a}-\lambda^{f}\right) /\left(\lambda^{a}+\lambda^{f}\right)$ is quantified by the dimensions $\lambda^{f, a}$ of the fore-aft segments, so that $p>0$ for $s^{+}$SPPs, $p<0$ for $s^{-}$SPPs, and $p=0$ for apolar rodlike SPPs. The effective aspect ratio is defined by $a=\ell / \lambda=1+$ $\varepsilon / 2$, with $\varepsilon \in[0.1,1]$ and $p \in[-0.8,0.8]$ in simulations.

The broken fore-aft symmetry results in fundamentally different collective behaviors of $s^{+}$SPPs and $s^{-}$SPPs, caused by their qualitatively different steric collision laws (Fig. 2) [34]. Polar $s^{+}$SPPs tend to align and experience only small speed changes during collisions, whereas antipolar $s^{-}$particles scatter broadly and experience a strong reduction of their speeds during the collision process [Figs. 2(a) and 2(b)]. On the mesoscopic level, these two collision scenarios translate into distinctly different patterns [Figs. 2(c) and 2(d)]. Polar $s^{+}$ SPPs form aligned large-scale swarms that move cooperatively along a spontaneously chosen common axis [27], whereas antipolar $s^{-}$SPPs tend to form droplets that nucleate slowly from an initially homogeneous suspension. Although this droplet formation may appear visually similar to chemotactic aggregation $[35,36]$, the underlying mechanism is purely steric and is intimately linked to an effectively density-dependent particle motility [29,37].

The clustering instability can be quantified in terms of the static structure factor, defined as $S(q)=\frac{1}{N}\left\langle\rho_{\mathbf{q}}(\tau) \rho_{-\mathbf{q}}(\tau)\right\rangle$, which is directly related to the number fluctuations in the limit of vanishing wave vector $q$ via $S(0)=\left(\left\langle N^{2}\right\rangle-\langle N\rangle^{2}\right) /\langle N\rangle$ [Figs. 2(e) and 2(f)]. In our simulations, we estimate $S(0) \approx$ $S\left(q_{\min }\right)$, where $q_{\min }=2 \pi / \sqrt{A / 4}$ is the smallest permissible wave number for a periodic simulation box of length $\sqrt{A}$. For $s^{-}$SPPs, a discontinuity in $S(0)$ with increasing $\phi$ marks the onset of the clustering instability at a volume filling fraction of $\phi \sim 0.05$ [Fig. 2(f)]. This transition is significantly weaker for the $s^{+}$SPPs [Fig. 2(e)]. In particular, plotting $S(0)$ as a function of polarity $p$ reveal that $p \approx-0.5$ is optimal for the self-assembly of clusters [Fig. 2(g)], corroborating that for convex polar SPPs the propensity to cluster depends crucially on the self-propulsion direction relative to the broken fore-aft symmetry. The differences in the collective behavior of $s^{+}$ SPPs and $s^{-}$SPPs become most prominent at intermediate packing fractions $\phi \sim 5 \%-10 \%$, a regime that can be achieved in suspensions of swimming microbes [38] and active colloids [39]. At very low or very high values of $\phi$, these differences vanish since the SPPs become effectively noninteracting (low $\phi)$ or too strongly hindered due to packing effects (large $\phi$ ).

To characterize in more detail the effects of shape on collective motions in homogeneous systems, we measured the mean speed $\langle v\rangle$ and the effective rotational diffusion coefficient $D_{r}=\lim _{\tau \rightarrow \infty}\left\langle[\Delta \varphi(\tau)]^{2}\right\rangle / 2 \tau$ from the mean-square angular deviation (MSAD) of the orientation angle $\varphi$. When

\footnotetext{
${ }^{1}$ The approximative representation of convex shapes by a finite number of spheres leads to small nonconvex intrusions [Fig. 1(e)], but these do not affect collective motion for the shape parameters used in our study. We verified in test simulations that finer discretizations with $n>3$ produce the same collective dynamics as those with $n=3$.
} 

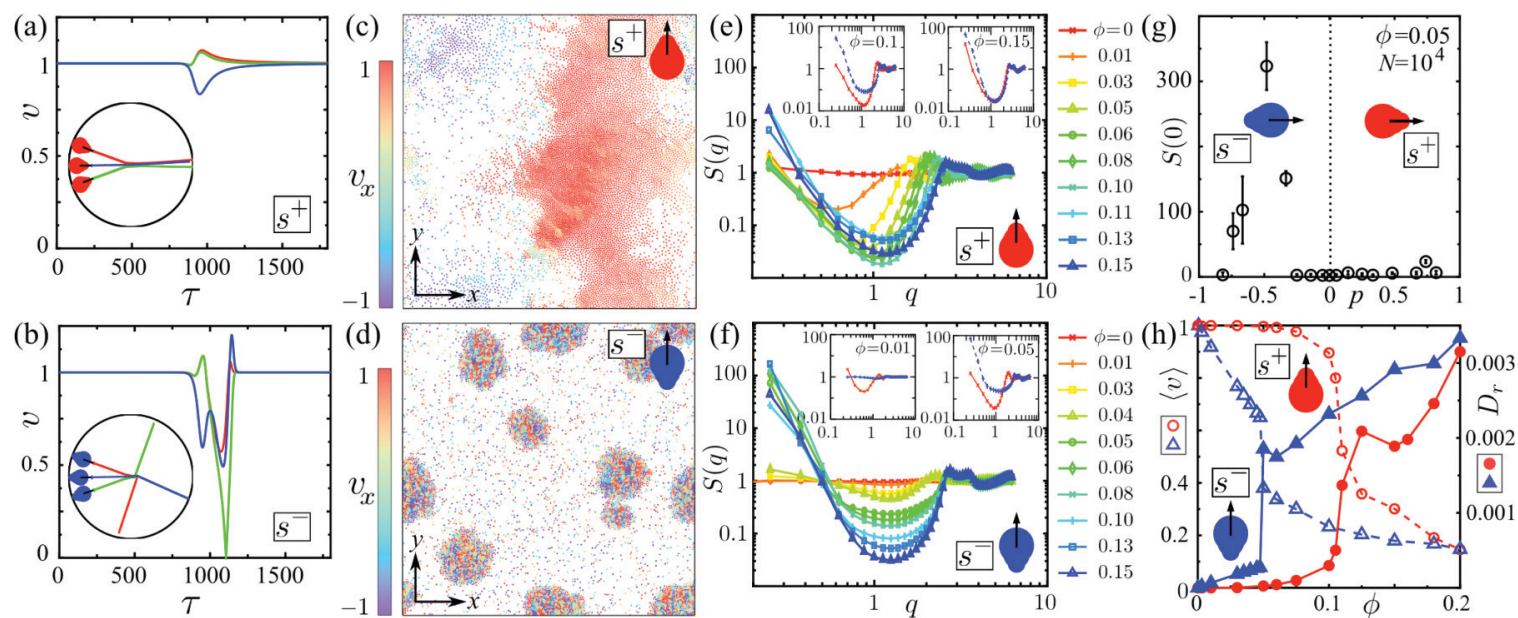

FIG. 2. (Color) Simulation results for uniform systems of convex particles [34]. (a) and (b) Time-dependent speed $v$ in units of $v_{0}$ and trajectories (insets) of three colliding (a) $s^{+}$SPPs and (b) $s^{-}$SPPs. (c) and (d) The $s^{+}$SPPs form aligned fronts, whereas $s^{-}$SPPs exhibit clustering. Color encodes the horizontal velocity component $v_{x}\left(N=10^{4},|p|=0.33\right.$, and $\left.\phi=0.05\right)$. (e) and (f) Static structure factor $S(q)$ at different volume filling fractions $\phi$. The insets show a comparison of $S(q)$ for $s^{+}$SPPs (red) and $s^{-}$SPPs (blue) at two different filling fractions $\phi$. (g) The peak of $S(0)$ at $p \approx-0.5$ indicates an optimal polarity for cluster formation. (h) Average velocity $\langle v\rangle$ for SPPs with $p= \pm 0.33$ (dashed lines with open symbols) and rotational diffusion coefficient $D_{r}$ of a tagged SPP (solid lines with closed symbols).

plotted as a function of the filling fraction $\phi$, both $\langle v\rangle$ and $D_{r}$ exhibit steep jumps for $s^{-}$while varying more smoothly for $s^{+}$ [Fig. 2(h)]. These jumps signal the onset of a nonequilibrium phase separation for $s^{-}$SPPs. A similar phenomenon was reported recently for a generalized Viscek model [40] and active Brownian spheres [41], suggesting this to be a generic feature of active systems with density-dependent mobility [37,39].

Demixing. The very different collective behaviors of $s^{+}$. and $s^{-}$SPPs suggest a novel shape-induced mechanism for demixing in active systems, which could be relevant for the segregation of species in microbiological systems. To test this idea, we simulated binary mixtures with equal numbers of $s^{+}$and $s^{-}$SPPs. These simulations show that such systems do indeed segregate into dense droplets of $s^{-}$SPPs that are almost completely devoid of $s^{+}$particles [Figs. 3(a) and 3(b)]. While demixing has generally been attributed to a strong disparity in particle motility [42], we find this phenomenon here in mixtures with identical single-particle motility.

The particle motions within the colonylike droplets exhibit clear signatures of dynamic heterogeneity, as $s^{-}$SPPs tend to move faster in the core of a droplet [Figs. 3(c) and 3(d)]. In contrast to the surrounding $s^{+}$swarms, the total net velocity of each $s^{-}$colony is very small, so they are virtually immobile. Moreover, we observe that $s^{+}$SPPs tend to accumulate at the outer regions of the droplets [Fig. 3(b)], reminiscent of bacterial cells accumulating on the surfaces of algal colonies. In addition to their potential biological implications, these findings demonstrate that mixtures of suitably shaped convex SPPs can provide a basis for the targeted self-assembly of active colloids in layers or shells.

Nonconvex SPPS. To explore the potential of another important classes of particle shapes for the self-assembly of active matter, we complement the above considerations by also discussing nonconvex particles, using crescent-shaped $c^{+}$SPPs and $c^{-}$anticrescents [Fig. 1(e)] as representative examples. Nonconvex self-propelled colloids were recently realized in experiments [20] and nonconvex shapes can also be found in various bacteria [22,43] [see Fig. 1(e)], but their collective behavior is yet to be studied systematically. In our simulations, we implemented crescent-shaped SPPs composed of $n=\lfloor 2 L / \lambda\rceil$ overlapping spherical segments (diameter $\lambda$ ), equidistantly spaced on a circular arc of fixed length $L=$ $2 \alpha R$. We quantify the degree of nonconvexity through the
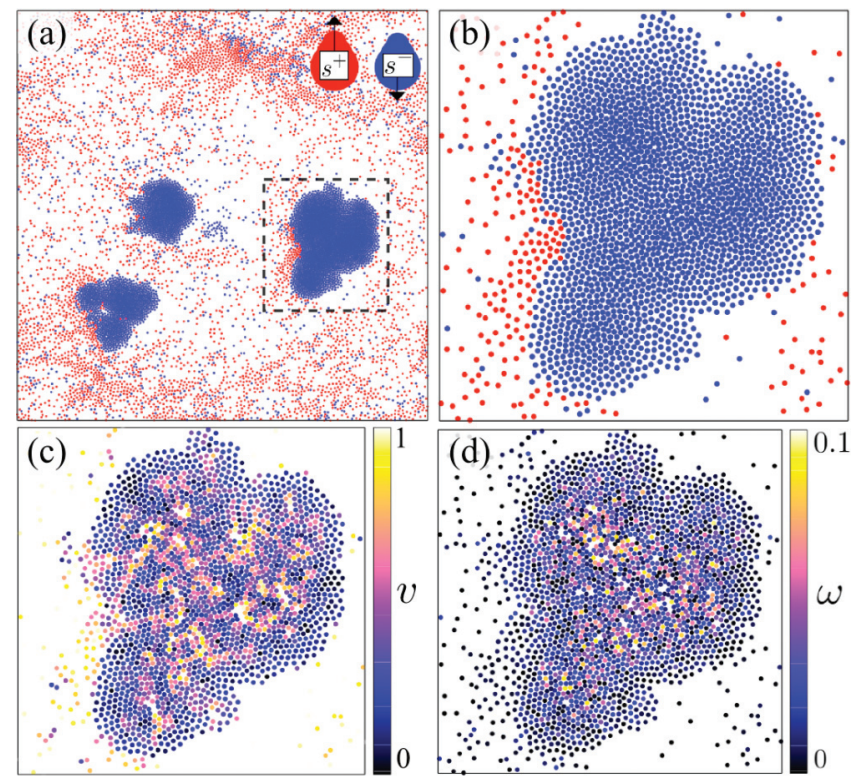

FIG. 3. (Color) Demixing of $s^{+}$SPPs and $s^{-}$SPPs in an equimolar binary suspension [34]. (a) Snapshots of the center-of-mass positions for $N=10^{4} \mathrm{SPPs}$ with $|p|=0.67$ and $\phi=0.05$. (b)-(d) Region enclosed by the dashed box in (a). Color in (c) and (d) encodes the translational speed $v$ and rotational velocity $\omega=|\partial \varphi / \partial \tau|$ of each SPP. 


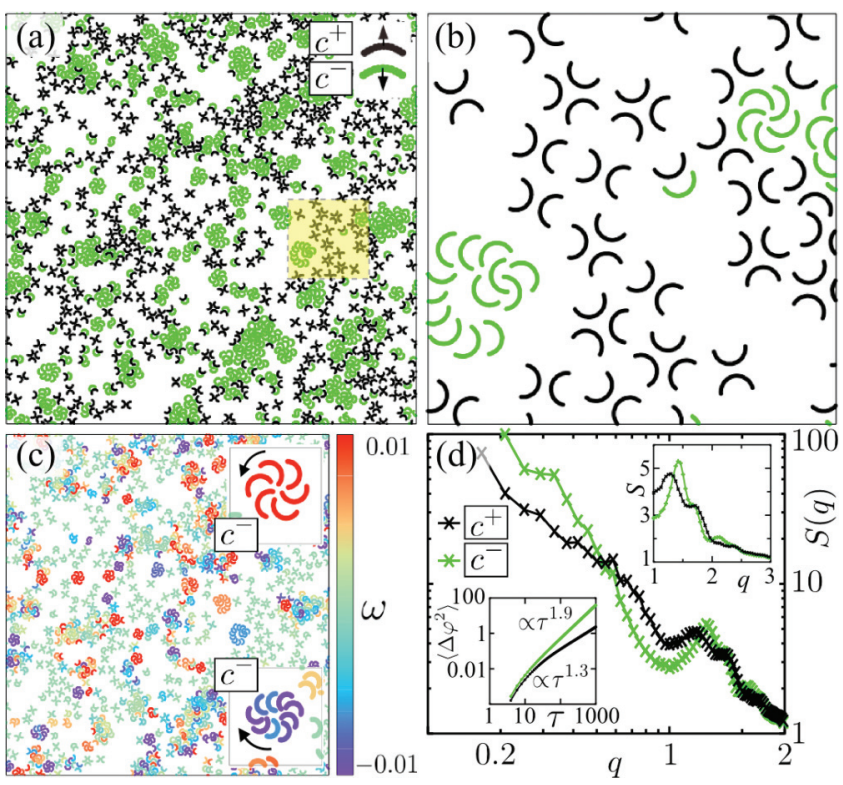

FIG. 4. (Color) Segregation and spontaneous self-assembly of $c^{-}$ rotors in an equimolar binary mixture of nonconvex SPPs [34]. (a) Snapshot of simulation with $N=2 \times 10^{3}, \kappa=0.2, \alpha=\pi$, and $\phi=$ 0.08. (b) Enlarged view of the yellow-shaded area in (a). (c) Rotational velocity $\omega=\partial \varphi / \partial \tau$ indicated by color coding. The insets depict snapshots of clusters composed of $c^{-}$crescents rotating clockwise $(\omega<0)$ or counterclockwise $(\omega>0)$. (d) A pronounced peak of the structure factor signals the formation of $c^{-}$rotors, which spin almost ballistically (lower left inset).

dimensionless curvature $\kappa=\lambda / R=2 \lambda \alpha / L$, defined such that straight rods are recovered in the limit $\alpha \rightarrow 0$ at constant arc length $L$. The effective aspect ratio $a=\ell / \delta$ of the $c^{ \pm}$ SPPs is determined by the dimensions $\ell=\lambda+L \sin \alpha / \alpha$ and $\delta=\lambda+L(1-\cos \alpha) / 2 \alpha$.

Similar to the convex case (Figs. 2 and 3), the trajectories of two or more colliding crescents depend sensitively on the swimming direction relative to the broken fore-aft symmetry, resulting in distinctly different mesoscale structures [Figs. 4(a) and 4(b)]. For a mixture containing equal numbers of $c^{ \pm}$SPPs, we again find segregation of the different particle types. More importantly, however, the $c^{-}$SPPs assemble into clockwise or counterclockwise spinning rotors [Figs. 4(a)-4(c)], characterized by a strongly superdiffusive (almost ballistic) collective rotational motion with MSAD $\left\langle(\Delta \varphi)^{2}\right\rangle \propto \tau^{\gamma}$, where $\gamma \approx 1.9$ [Fig. 4(d)]. By contrast, the $c^{+}$SPPs very rarely cluster beyond pairs or triplets, exhibiting only weakly superdiffusive rotational motion with exponent $\gamma \approx 1.3$. Test simulations showed that $c^{-}$rotors are robust against thermal fluctuations, whereas the small $c^{+}$clusters decay rapidly in the presence of noise. Generally, this basic example illustrates how subtle differences in curvature that break fore-aft symmetry, combined with self-propulsion, can be exploited to self-assemble microrotors [30,31,44] from linearly moving nonconvex objects.

Discussion and conclusions. We have used large-scale 2D SPP simulations to investigate how particle symmetry affects active collective motion at surfaces and interfaces. Self-propelled particle simulations have proven useful in the past for understanding generic aspects of collective behavior in bacterial suspensions [12] and other active systems [42,45-48], but such earlier studies focused almost exclusively on fore-aft symmetric (e.g., spherical or rodlike) particles [28]. Our results show that even subtle violations of fore-aft symmetry can lead to profound changes in the collective dynamics of active colloids or micro-organisms, promising new strategies for the self-assembly of active matter. The systematic classification of macroscopic material properties in terms of microscopic constituent symmetries [1-3,9] has been very fruitful for the understanding of conventional passive matter. We therefore believe that, with regard to future applications, it is worthwhile to continue exploring more systematically, both theoretically and experimentally, the interplay between geometric particle symmetries and self-propulsion in active systems.

From a theoretical perspective, simplified shape-based models can provide a useful conceptual link between abstract Viscek-type vector-based theories [40,49,50] and more accurate microscopic models [51]. With regard to microbiological systems, the usefulness of such geometric approaches depends on the collision time scale of the organisms, which determines whether time-averaged shapes, as shown in Figs. 1(b) and 1(c), are sufficiently accurate approximations to the most relevant interaction effects. However, recent experiments on microbial surface interactions [26], as well as the results in Figs. 2 and 3, suggest that shape-induced effects, in conjunction with chemical sensing and hydrodynamic effects, could indeed be a relevant factor in microbial processes such as collective sperm swimming or algal colony formation. Finally, in view of the current rapid progress in the fabrication of active colloids [20] and in the manipulation of microbial shapes [22,52], we expect that the above results can provide useful guidance for the controlled self-assembly of active (e.g., layered or rotating) mesoscale structures in the near future.

Acknowledgments. This work was supported by the ERC Advanced Investigator Grant No. 247333 (R.E.G.). The authors thank H. Chaté for helpful comments. The image in Fig. 1(a) was kindly provided by F. Kümmel and C. Bechinger. The image in Fig. 1(d) was reprinted with kind permission from Ref. [43].
[1] L. Pauling, J. Am. Chem. Soc. 51, 1010 (1929).

[2] P. F. Damasceno, M. Engel, and S. C. Glotzer, Science 337, 453 (2012).

[3] J. de Graaf and L. Manna, Science 337, 417 (2012).

[4] T. Gibaud et al., Nature (London) 481, 348 (2012).

[5] V. Schaller and A. R. Bausch, Nature (London) 481, 268 (2012).
[6] A. Hirsch, Nat. Mater. 9, 868 (2010).

[7] W. 1. Mao et al., Science 302, 425 (2003).

[8] L. Wang, B. Liu, H. Li, W. Yang, Y. Ding, S. V. Sinogeikin, Y. Meng, Z. Liu, X. C. Zeng, and W. L. Mao, Science 337, 825 (2012).

[9] CEGEO, B. Saint-Cyr et al., Eur. Phys. Lett. 98, 44008 (2012). 
[10] S. Ramaswamy, Annu. Rev. Condens. Matter Phys. 1, 323 (2010).

[11] A. M. Menzel and T. Ohta, Eur. Phys. Lett. 99, 58001 (2012).

[12] H. H. Wensink, J. Dunkel, S. Heidenreich, K. Drescher, R. E. Goldstein, H. Löwen, and J. M. Yeomans, Proc. Natl. Acad. Sci. U.S.A. 109, 14308 (2012).

[13] E. Lauga and R. E. Goldstein, Phys. Today 65(9), 30 (2012).

[14] T. Brotto, J.-B. Caussin, E. Lauga, and D. Bartolo, Phys. Rev. Lett. 110, 038101 (2013).

[15] J. Dunkel, S. Heidenreich, K. Drescher, H. H. Wensink, M. Bär, and R. E. Goldstein, Phys. Rev. Lett. 110, 228102 (2013).

[16] T. Hanke, C. A. Weber, and E. Frey, Phys. Rev. E 88, 052309 (2013).

[17] J. A. Champion, Y. K. Katare, and S. Mitragotri, J. Control. Release 121, 3 (2007).

[18] D. Rodriguez-Fernandez and L. M. Liz-Marzan, Part. Part. Syst. Char. 30, 46 (2013).

[19] D. Takagi, A. B. Braunschweig, J. Zhang, and M. J. Shelley, Phys. Rev. Lett. 110, 038301 (2013).

[20] F. Kümmel, B. ten Hagen, R. Wittkowski, I. Buttinoni, R. Eichhorn, G. Volpe, H. Löwen, and C. Bechinger, Phys. Rev. Lett. 110, 198302 (2013).

[21] K. D. Young, Microbiol. Mol. Biol. Rev. 70, 660 (2006).

[22] M. T. Cabeen, G. Charbon, W. Vollmer, P. Born, N. Ausmees, D. B. Weibel, and C. Jacobs-Wagner, EMBO J. 28, 1208 (2009).

[23] A. Be'er, S. K. Strain, R. A. Hernandez, E. Ben-Jacob, and E. Florin, J. Bacteriol. 195, 2907 (2013).

[24] K. Drescher, J. Dunkel, L. H. Cisneros, S. Ganguly, and R. E. Goldstein, Proc. Natl. Acad. Sci. U.S.A. 108, 10940 (2011).

[25] I. Aronson, Physics 6, 61 (2013).

[26] V. Kantsler, J. Dunkel, M. Polin, and R. E. Goldstein, Proc. Natl. Acad. Sci. U.S.A. 110, 1187 (2013).

[27] G. Grégoire and H. Chaté, Phys. Rev. Lett. 92, 025702 (2004).

[28] C. A. Weber, T. Hanke, J. Deseigne, S. Léonard, O. Dauchot, E. Frey, and H. Chaté, Phys. Rev. Lett. 110, 208001 (2013).

[29] Y. Fily and M. C. Marchetti, Phys. Rev. Lett. 108, 235702 (2012).

[30] A. Sokolov, M. M. Apodacac, B. A. Grzybowskic, and I. S. Aranson, Proc. Natl. Acad. Sci. U.S.A. 107, 969 (2009).

[31] R. Di Leonardo et al., Proc. Natl. Acad. Sci. U.S.A. 107, 9541 (2010).
[32] E. M. Purcell, Am. J. Phys. 45, 3 (1977).

[33] M. M. Tirado, J. G. de la Torre, and C. L. Martinez, J. Chem. Phys. 81, 2047 (1984).

[34] See Supplemental Material at http://link.aps.org/supplemental/ 10.1103/PhysRevE.89.010302 for movies of the collective SPP dynamics.

[35] M. J. Tindall, P. K. Maini, S. L. Porter, and J. P. Armitage, Bull. Math. Biol. 70, 1570 (2008).

[36] P. Romanczuk, U. Erdmann, H. Engel, and L. SchimanskyGeier, Eur. Phys. J. Spec. Top. 157, 61 (2008).

[37] J. Tailleur and M. E. Cates, Phys. Rev. Lett. 100, 218103 (2008).

[38] A. Sokolov and I. S. Aranson, Phys. Rev. Lett. 109, 248109 (2012).

[39] I. Buttinoni, J. Bialké, F. Kümmel, H. Löwen, C. Bechinger, and T. Speck, Phys. Rev. Lett. 110, 238301 (2013).

[40] F. D. C. Farrell, M. C. Marchetti, D. Marenduzzo, and J. Tailleur, Phys. Rev. Lett. 108, 248101 (2012).

[41] G. S. Redner, M. F. Hagan, and A. Baskaran, Phys. Rev. Lett. 110, 055701 (2013).

[42] S. R. McCandlish, A. Baskaran, and M. F. Hagan, Soft Matter 8, 2527 (2012).

[43] K. Zhang and X. Dong, Int. J. Syst. Evol. Microbiol. 59, 2080 (2009).

[44] J. Schwarz-Linek, C. Valeriani, A. Cacciuto, M. E. Cates, D. Marenduzzo, A. N. Morozov, and W. C. K. Poon, Proc. Natl. Acad. Sci. U.S.A. 109, 4052 (2012).

[45] F. Peruani, A. Deutsch, and M. Bär, Phys. Rev. E 74, 030904 (2006).

[46] A. Baskaran and M. C. Marchetti, Phys. Rev. E 77, 011920 (2008).

[47] H. H. Wensink and H. Löwen, J. Phys.: Condens. Matter 24, 464130 (2012).

[48] E. Lushi and C. S. Peskin, Comput. Struct. 122, 239 (2013).

[49] T. Vicsek, A. Czirók, E. Ben-Jacob, I. Cohen, and O. Shochet, Phys. Rev. Lett. 75, 1226 (1995).

[50] P. Romanczuk, M. Bär, W. Ebeling, B. Lindner, and L. Schimansky-Geier, Eur. Phys. J. Spec. Top. 202, 1 (2012).

[51] S. B. Babu and H. Stark, New J. Phys. 14, 085012 (2012).

[52] A. Amir, F. Babaeipour, D. R. Nelson, and S. Jun, arXiv:1305.5843. 